\title{
Levels of CMV-reactive antibodies correlate with the induction of CD28null T-cells and systemic inflammation in chronic obstructive pulmonary disease (COPD)
}

Dino BA Tan ${ }^{1,2}$, Fathiah S. Amran ${ }^{3}$, Teck-Hui Teo ${ }^{3}$, Patricia Price ${ }^{3,4}$, Yuben P. Moodley ${ }^{1,2,5}$

${ }^{1}$ Centre for Asthma, Allergy and Respiratory Research, School of Medicine \& Pharmacology, University of Western Australia, Nedlands, WA, Australia

${ }^{2}$ Stem Cell Unit, Lung Institute of WA, University of Western Australia, Nedlands, WA, Australia

${ }^{3}$ School of Pathology and Laboratory Medicine, University of Western Australia, Nedlands, WA, Australia

${ }^{4}$ School of Biomedical Science, Curtin University, Bentley, WA, Australia

${ }^{4}$ Department of Respiratory and Sleep Medicine, Royal Perth Hospital, Perth, WA, Australia

\section{Corresponding author:}

Dr Dino Bee Aik Tan

Level 2, Medical Research Foundation Building

Royal Perth Hospital,

Rear 50 Murray Street,

Perth, WA 6000, Australia

Email: dino.tan@uwa.edu.au

Phone: +61892240270

Keywords: CMV, COPD, CD28null T-cell, chronic inflammation 
Chronic obstructive pulmonary disease (COPD) is characterized by chronic irreversible inflammation and progressive decline in lung function. While smoking and other environmental agents (e.g. air pollutants) are common causes of COPD, the major challenges in managing this condition are the persistent inflammation and functional deterioration which are not reversed by removal of precipitating agents (e.g. smoking cessation) or treatment with anti-inflammatory agents. We hypothesized that the reactivation of latent CMV infection may trigger expansion of proinflammatory CD28null T-cells and hence promote sysmetic inflammation in patients with COPD. This study presents novel data showing increased levels of CMV-reactive IgM and IgG in COPD patients compared to controls. This paralleled observed increases in markers of sytemic inflammation ex vivo and proportions of circulating CD28null T-cells. This mechanism may explain the persistent inflammation in COPD patients.

COPD is a leading cause of global mortality and morbidity which affects mostly smokers and the elderly. It involves airway and systemic inflammation and progressive decline in lung function due to airway damage and airflow obstruction. Acute exacerbations of COPD (AECOPD), defined as significant worsening of COPD symptoms requiring treatment modifications and/or hospitalization, contribute to functional deterioration and heightened inflammation ${ }^{1}$.

Acute respiratory viral infections (e.g. influenza) are commonly associated with AECOPD, and latent adenoviral infections have also been implicated ${ }^{2}$. However no studies have addressed the roles of herpes viruses such as cytomegalovirus (CMV) that persist in a latent state and can be reactivated by inflammation or immunosuppression. CMV infections are usually asymptomatic in healthy individuals but can cause life-threatening end-organ disease in immune-compromised individuals (e.g. transplant recipients or HIV patients). Whilst symptomatic CMV diseases are rare in COPD patients, chronic inflammation or treatment with corticosteroids may promote CMVrelated complications including pneumonitis and esophagitis ${ }^{3}$. 
As COPD is a disease of old age, it is valid to consider how it may be affected by the impact of repeated CMV reactivations on the immune system. Furthermore, both COPD and CMV replication increase the incidence of age-associated systemic co-morbidities (e.g. cardiovascular diseases) and are associated with expanded populations of circulating CD28null T-cells (marker of immune senescence/aging) that are pro-inflammatory ${ }^{1,4-7}$. Therefore, we investigated the link between COPD and levels of CMV-reactive antibodies in the context of chronic inflammation and T-cell senescence.

The study was approved by the Ethics Committee at the Royal Perth Hospital and all participants gave informed consent. We recruited AECOPD patients $(n=21)$ who were admitted for exacerbation to the Royal Perth Hospital Emergency Department (Western Australia) and stable COPD (sCOPD) patients $(n=33)$ who were previous smokers ( $>15$ pack-years and ceased smoking $>5$ years earlier) from a dedicated COPD clinic at Royal Perth Hospital. The diagnosis and severity of COPD were established using the Global Initiative for Chronic Obstructive Lung Disease guidelines (Stages 24). All patients had been treated with anticholinergic drugs, long-acting beta agonists and inhaled corticosteroids but none were receiving systemic corticosteroids or had diabetes, neuromuscular, allergic or rheumatological disease. Healthy age-matched non-smoking controls with no clinical evidence of COPD were included $(n=33)$.

Levels of IgG and IgM reactive with a lysate of CMV-infected fibroblasts (CMV-lysate), CMV glycoprotein-B (CMV-gB) and CMV immediate early-1 antigen (CMV-IE-1) in plasma were measured by in-house ELISAs ${ }^{8}$. Three antigen preparations of CMV were used in our assay because CMV-lysate contains several CMV antigens, whilst antibodies to CMV-IE-1 arise during early CMV infection and antibodies against CMV-gB denotes previous infection. Plasma levels of soluble tumour necrosis factor receptor-1 (sTNFR1), a marker of systemic inflammation, were measured using a commercial ELISA kit (R\&D Systems, Minneapolis, MN, USA) ${ }^{8}$. Proportions of 
$\mathrm{CD} 28$ null $\mathrm{CD} 4^{+}$and $\mathrm{CD} 8^{+} \mathrm{T}$-cells were quantified by flow cytometry after staining peripheral blood mononuclear cells with anti-CD3-APC-H7, CD4-V500, CD8-PerCP-Cy5.5 and CD28-PE-Cy7 (BD Biosciences, San Jose, CA, USA).

Levels of $\operatorname{IgM}$ and $\operatorname{IgG}$ reactive with CMV-lysate were higher in AECOPD ( $\mathrm{p}=0.022 \& 0.02$ respectively) and sCOPD patients (0.002 \& 0.014, respectively) than controls, but did not differ between AECOPD and sCOPD patients (Figure 1A \& 1B). Similar trends were observed with titers of IgG reactive with CMV-gB (Figure 1C), whilst IgG reactive with CMV-IE-1 were similar between groups (Figure 1D). There were higher proportions of CMV sero-negative individuals amongst controls than patients $(24 \%=5 / 21$ AECOPD; $21 \%=7 / 33 \mathrm{sCOPD} ; 36 \%=12 / 33$ controls $)$. These individuals had $<300 \mathrm{U} / \mathrm{mL}$ IgG (below the broken line; Figure 1B), using a cut-off defined by 11 individuals deemed CMV sero-negative using a commercial microparticle enzyme immunoassay (Abbott Laboratories, IL, USA). After the exclusion of CMV sero-negative subjects, levels of IgG reactive with CMV-lysate were still higher in AECOPD and sCOPD patients ( $\mathrm{p}=0.003$ $\& 0.02$, respectively) than controls.

AECOPD and sCOPD patients exhibited higher levels of sTNFR1 than controls $(\mathrm{p}<0.001$ and 0.007, respectively). Levels of sTNFR1 were also higher in AECOPD than sCOPD patients ( $\mathrm{p}=0.016$; Figure 1E). Levels of IgM and IgG reactive with CMV antigens correlated with sTNFR1 levels $(\mathrm{r}=0.25-0.28, \mathrm{p}=0.006-0.02)$, but not with the level of total $\mathrm{IgG}$ or lung function (data not shown). The plasma concentration of total IgG was lower in AECOPD than controls $(\mathrm{p}=0.018)$, but similar to controls in $\mathrm{sCOPD}$ patients $(\mathrm{p}=0.81$; Figure $1 \mathrm{~F})$. Therefore, elevation of CMV-reactive antibodies was specific and did not reflect B-cell activation because total IgG levels were not elevated in COPD patients. 
CMV reactivation has been associated with chronic inflammation ${ }^{4}$. In a murine model, inflammatory cytokines (e.g. TNF) or allogeneic transplantation induced CMV reactivations ${ }^{9}$. Inflammation in COPD patients (triggered by precipitates including tobacco and air pollution) may promote CMV reactivation thus perpetuating low grade inflammation. In this study, the smoking history of COPD patients (pack-years) correlated positively with CMV-reactive antibody ( $\mathrm{r}=0.35$ 0.46, $\mathrm{p}=0.004-0.03)$. The correlation between smoking history and the level of CMV-reactive antibodies suggests an interaction of these two inflammatory stimuli in the pathogenesis of COPD.

Proportions of $\mathrm{CD} 28$ null $\mathrm{CD}^{+}$and $\mathrm{CD}^{+} \mathrm{T}$-cells were higher in sCOPD patients than controls $(\mathrm{p}=0.02 \& 0.005$, respectively) (Figure $1 \mathrm{G} \& 1 \mathrm{H})$. The proportions of CD28null $\mathrm{CD} 4^{+}$and $\mathrm{CD} 8^{+} \mathrm{T}-$ cells correlated with levels of $\operatorname{IgG}$ reactive with CMV-lysate $(r=0.48, p<0.001 \& r=0.42, p=0.001$, respectively), CMV-gB $(\mathrm{r}=0.42, \mathrm{p}=0.001 \& \mathrm{r}=0.34, \mathrm{p}=0.01$, respectively) and CMV-IE-1 $(\mathrm{r}=0.43$, $\mathrm{p}<0.001 \& \mathrm{r}=0.31, \mathrm{p}=0.02$, respectively).

The loss of CD28 expression on T-cells reflects immune aging arising from chronic antigen stimulation. These senescent T-cells have reduced telomere length, aberrant DNA methylation and overexpression of pro-inflammatory genes ${ }^{7}$. $\mathrm{CD} 28$ null $\mathrm{CD}^{+}$and $\mathrm{CD}^{+}$T-cells have been implicated in COPD and autoimmune diseases ${ }^{5,6}$. 10 In COPD, CD28null T-cells produce more interferon- $\gamma$, granzyme-B and perforin than $\mathrm{CD} 28+\mathrm{CD}^{+}$T-cells ${ }^{5}$. Smokers without COPD showed no increase in $\mathrm{CD} 28$ null $\mathrm{CD} 4^{+} \mathrm{T}^{-}$-cells ${ }^{5}$. In patients with multiple sclerosis and rheumatoid arthritis, CD28null T-cells were mostly reactive with CMV antigens rather than self-antigens and were resistant to suppressive action of regulatory $\mathrm{T}$-cells ${ }^{10}$. This suggests that the accumulation of CD28null T-cells in COPD is more likely due to CMV reactivation than smoking, thus promoting chronic inflammation and further enhancing both diseases. 
In conclusion, our study supports a role for CMV in the pathogenesis of COPD. Reactivation of CMV may trigger the induction of CD28null T-cells and promote systemic inflammation characteristic of COPD patients. Future studies investigating the role of T-cells in COPD should consider the CMV sero-status of patients since CMV plays a role in mediating chronic inflammation and augmenting the function of inflammatory T-cells. 


\section{Acknowledgements}

This work was supported by an Ada Bartholomew Grant from The University of Western Australia and a MRF Young Investigator Grant from Royal Perth Hospital. The authors would like to thank the staff at the Centre for Clinical Research in Emergency Medicine, Harry Perkins Institute of Medical Research and the COPD Linkage clinic, Royal Perth Hospital for patient recruitment, and the patients and controls who volunteered for this study. 


\section{Figure legend}

Figure 1 - Plasma levels of CMV-reactive IgM and IgG, sTNFR1 and total IgG and circulating proportions of CD28null T-cells in patients with AECOPD and SCOPD compared with healthy controls. The levels of IgM against CMV-lysate (A), IgG against CMV-lysate (B), IgG against CMV-gB (C), IgG against CMV-IE-1 (D), sTNFR1 (E) and total IgG (F) in plasma were measured by ELISA. Circulating proportions of $\mathrm{CD} 28$ null $\mathrm{CD}^{+}(\mathrm{G})$ and $\mathrm{CD}^{+} \mathrm{T}$-cells $(\mathrm{H})$ were quantified by flow cytometry. Non-parametric Mann-Whitney test was used to compare the medians between AECOPD, sCOPD and healthy controls using the Prism 5.04 (GraphPad Software, La Jolla, CA). Horizontal lines denote median and only significant $p$-values $(\mathrm{p}<0.05)$ were presented on graphs. 


\section{References}

1. Barnes PJ and Celli BR. Systemic manifestations and comorbidities of COPD. Eur Respir J 2009. 33: 1165-1185.

2. Hogg JC. Role of latent viral infections in chronic obstructive pulmonary disease and asthma. Am J Respir Crit Care Med 2001. 164: S71-75.

3. Weile J, Streeck B, Muck J, Krebs G, Jakobus KH, Knabbe C et al. Severe cytomegalovirusassociated esophagitis in an immunocompetent patient after short-term steroid therapy. $J$ Clin Microbiol 2009. 47: 3031-3033.

4. Simanek AM, Dowd JB, Pawelec G, Melzer D, Dutta A, and Aiello AE. Seropositivity to cytomegalovirus, inflammation, all-cause and cardiovascular disease-related mortality in the United States. PLoS One 2011. 6: e16103.

5. Lambers C, Hacker S, Posch M, Hoetzenecker K, Pollreisz A, Lichtenauer M et al. T cell senescence and contraction of $\mathrm{T}$ cell repertoire diversity in patients with chronic obstructive pulmonary disease. Clin Exp Immunol 2009. 155: 466-475.

6. Hodge G, Mukaro V, Reynolds PN, and Hodge S. Role of increased CD8/CD28(null) T cells and alternative co-stimulatory molecules in chronic obstructive pulmonary disease. Clin Exp Immunol 2011. 166: 94-102.

7. Moro-Garcia MA, Alonso-Arias R, and Lopez-Larrea C. Molecular mechanisms involved in the aging of the T-cell immune response. Curr Genomics 2012. 13: 589-602.

8. Brunt SJ, Lee S, D'Orsogna L, Bundell C, Burrows S, and Price P. The Use of Humoral Responses as a Marker of CMV Burden in HIV Patients on ART Requires Consideration of TCell Recovery and Persistent B-Cell Activation. Disease Markers 2014. 2014: 8.

9. Hummel M and Abecassis MM. A model for reactivation of CMV from latency. J Clin Virol 2002. 25 Suppl 2: S123-136. 
10. Thewissen M, Somers V, Hellings N, Fraussen J, Damoiseaux J, and Stinissen P. CD4+CD28null $\mathrm{T}$ cells in autoimmune disease: pathogenic features and decreased susceptibility to immunoregulation. J Immunol 2007. 179: 6514-6523. 
